\title{
AVALIAÇÃO DA EFICIÊNCIA DA ELETROCOAGULAÇÃO- FLOTAÇÃ̃O NA DESCOLORAÇÃO DE EFLUENTE TÊXTIL
}

\author{
J. E. C. ALEXANDRE ${ }^{1}$, T. C. PARENTE ${ }^{2}$, J. P. RIBEIRO ${ }^{1}$, L. G.C. MAIA ${ }^{1}$, E. F. ABDALA NETO ${ }^{1}$ \\ e R. F. do NASCIMENTO ${ }^{3}$ \\ ${ }^{1}$ Universidade Federal do Ceará, Departamento de Engenharia Hidráulica e Ambiental \\ ${ }^{2}$ Universidade Federal do Ceará, Departamento de Engenharia Química \\ ${ }^{3}$ Universidade Federal do Ceará, Departamento de Química Analítica \\ E-mail para contato: jessicaelen.alexandre@gmail.com
}

\begin{abstract}
RESUMO - O tratamento de efluente têxtil é uma prática extremamente necessária, principalmente se o destino final for o descarte, tendo em vista o alto volume gerado, bem como sua composição. A eletrocoagulação-flotação tem se destacado dentre as técnicas não convencionais de tratamento destes efluentes pela perspectiva de elevada eficiência operacional, principalmente atuando na degradação das moléculas dos corantes. O presente trabalho visa avaliar a eficiência da descoloração de um efluente real têxtil utilizando eletrodos de alumínio. Os experimentos foram conduzidos em um reator de eletrocoagulação-flotação, cilíndrico de 2,7 L, operando em batelada. Variou-se a velocidade de agitação em 0, 200 e $700 \mathrm{rpm}$ e foram monitorados: densidade de corrente, temperatura, $\mathrm{pH}$, turbidez e absorbância no UV-visível. Os resultados obtidos mostraram redução de turbidez em torno de $96 \%$ e significativo decaimento da absorbância na faixa do UV-vis, atestando a descoloração final do efluente tratado, em apenas 10 minutos de eletrólise e sem a necessidade de agitação.
\end{abstract}

\section{INTRODUÇÃO}

A agressividade ao meio ambiente vem aumentando progressivamente, à medida que ocorre o acelerado crescimento populacional e o aumento da atividade industrial. Dentro deste contexto, o setor têxtil apresenta um especial destaque, devido à elevada demanda de água exigida em seus processos e a consequente geração de grandes volumes de efluentes. Estima-se que na produção de tecido de algodão, por exemplo, o consumo de água pode variar de 100 a $300 \mathrm{~L} . \mathrm{Kg}^{-1}$ de tecido. (MEDEIROS, 2011).

Os efluentes têxteis caracterizam-se por serem altamente coloridos, devido à presença de corantes que não se fixam na fibra durante o processo de tingimento, e quando lançados sem um tratamento adequado podem provocar graves problemas para a vida aquática, como também para a saúde humana. Os corantes sintéticos são moléculas orgânicas complexas de difícil degradação biológica, apresentando, pois, elevada recalcitrância (HASSEMER e SENS, 2002). 
Atualmente, vários processos estão sendo extensivamente utilizados para o tratamento de efluentes têxteis e dentre eles podem ser mencionados: a adsorção, a precipitação, a coagulação, a biodegradação, a degradação química e os processos de oxidação avançada envolvendo técnicas eletroquímicas. Oportunamente, a eletrocoagulação-flotação vem despertando o interesse do meio científico por se tratar de uma tecnologia versátil, de simples aplicação, além de não requerer adição de qualquer reagente químico.

No processo de eletrocoagulação-flotação (ECF), o coagulante provém dos eletrodos utilizados ocasionando a neutralização das cargas superficiais das partículas coloidais e suspensas, desestabilizando-as (eletrocoagulação). A aglutinação das partículas desestabilizadas pelos hidróxidos metálicos proporciona então, o crescimento dos flocos (eletrofloculação). Simultaneamente aos processos descritos anteriormente, as micro-bolhas de oxigênio $\left(\mathrm{O}_{2}\right)$ geradas no ânodo e de hidrogênio $\left(\mathrm{H}_{2}\right)$ geradas no cátodo, formadas pela hidrólise da água ascendem à superfície carregando, por arraste, os flocos formados pelas impurezas e assim promovendo a clarificação do efluente (eletroflotação). A duração do processo de flotação é marcantemente mais curta comparada ao de sedimentação depois da coagulação. (HU et al., 2005).

No processo ECF utilizando eletrodos de alumínio, as principais reações de eletrólise que ocorrem encontram-se a seguir, representadas pelas as equações de 1 a 3:

A liberação de íons de $\mathrm{Al}^{3+}$ :

$$
A l \rightarrow A l^{3+}+3 e^{-}(\hat{A} \text { nodo })
$$

A oxidação da água:

$$
2 \mathrm{H}_{2} \mathrm{O} \rightarrow 4 \mathrm{H}^{+}+\mathrm{O}_{2}+4 e^{-} \text {(Ânodo) }
$$

A redução da água:

$$
2 \mathrm{H}_{2} \mathrm{O}+2 e^{-} \rightarrow \mathrm{H}_{2}+2 \mathrm{OH}^{-} \text {(Cátodo) }
$$

Como também ocorre a reação de formação do hidróxido de alumínio através da reação entre os íons $\mathrm{Al}^{3+}$ e $\mathrm{OH}^{-}$gerados nas superfícies dos eletrodos, representada a seguir pela equação 4:

$$
A l^{3+}+3 O H^{-} \rightarrow A l(O H)_{3}
$$

Diante do exposto, o presente trabalho visa avaliar a eficiência da eletrocoagulação-flotação por corrente alternada, utilizando eletrodos de alumínio para a descoloração de um efluente têxtil proveniente de uma indústria de redes. 


\section{METODOLOGIA}

\subsection{O sistema de ECF}

O processo eletrolítico foi conduzido em um reator cilíndrico (diâmetro $=10 \mathrm{~cm}$; altura $=$ $60 \mathrm{~cm}$ ) de acrílico, conforme apresentado na Figura 1, operando em batelada. O conjunto de eletrodos foi construído com 4 placas de alumínio, conectadas por um arranjo bipolar, cada uma medindo $5 \times 40 \times 0,3 \mathrm{~cm}$, resultando numa área total de $800 \mathrm{~cm}^{2}$. Os eletrodos foram dispostos com o espaçamento de $1 \mathrm{~mm}$ entre si. A promoção da eletrólise foi realizada por meio de uma fonte retificadora de corrente elétrica da marca Hayama ${ }^{\circledR} 220 \mathrm{~V}-13,8 \mathrm{~V}$ e de forma não convencional, utilizando-se um conversor CC/CA, promovendo corrente alternada nos eletrodos metálicos submersos, gerando ondas eletromagnéticas, com número de onda entre 0 e $4000 \mathrm{~cm}^{-1}$ (contemplando o espectro do infravermelho). Para os experimentos em que a agitação foi requerida, utilizou-se um agitador magnético fabricado pela Tecnal ${ }^{\circledR} \mathrm{TE}-0851^{\mathrm{TM}}$.

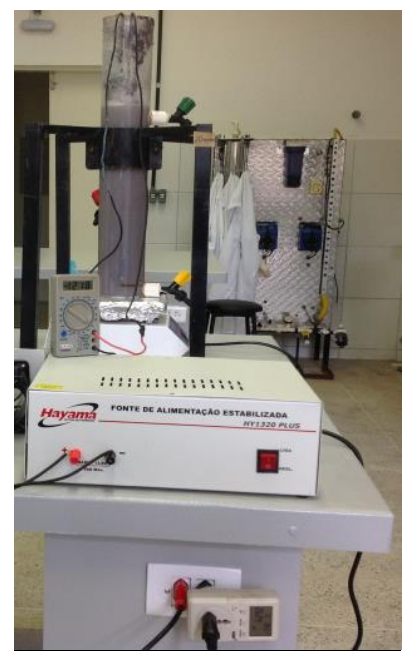

Figura 1 - O sistema de ECF.

\subsection{Ensaios de descoloração/degradação e técnicas analíticas}

Para a realização dos ensaios de ECF, foi utilizado o volume de $2,7 \mathrm{~L}$ de efluente têxtil, e variou-se a velocidade de agitação em 0,200 e $700 \mathrm{rpm}$, com a finalidade de estudar a influência da mesma no processo. Os experimentos em bateladas foram realizados com os seguintes tempos pré-estabelecidos: $1,3,5,7,10,20$ e 30 min, sendo monitorados densidade de corrente (mA.cm ${ }^{-}$ ${ }^{2}$ ), turbidez (NTU), cor, $\mathrm{pH}$ e temperatura $\left({ }^{\circ} \mathrm{C}\right)$. É importante destacar que, o termopar de leitura da temperatura foi posicionado, para todos os experimentos, a $20 \mathrm{~cm}$ do topo do reator.

A turbidez (NTU) foi determinada pelo método nefelométrico em um turbidímetro portátil da $\operatorname{Hach}^{\circledR} 2100 \mathrm{P}^{\mathrm{TM}}$. A descoloração foi avaliada através da medição da absorbância no 
espectrofotômetro Shimadzu ${ }^{\circledR} \mathrm{UV}-1800^{\mathrm{TM}}$ na região de $230-750 \mathrm{~nm}$. O valor do pH foi determinado utilizando um pHmetro da Tecnal ${ }^{\circledR}$ Tec $5{ }^{\mathrm{TM}}$. A medida da condutividade foi realizada com um condutivímetro Hanna ${ }^{\circledR}, \mathrm{HI} 4321^{\mathrm{TM}}$. Todas as análises foram executadas conforme $\mathrm{o}$ Standard Methods for Examination of Water and Wastewater (APHA, 2005).

\section{RESULTADOS E DISCUSSÃO}

\subsection{Caracterização do efluente bruto}

Os resultados encontrados da caracterização físico-química do efluente bruto foram: 8,7 para o pH, 140 NTU para a turbidez, $13,66 \mathrm{mS} / \mathrm{cm}$ para a condutividade e $4511 \mathrm{mg} / \mathrm{L}$ para a concentração de íon cloreto. É importante ressaltar que este valor considerável de condutividade, decorrente da alta concentração de sal existente no efluente, favorece o processo de ECF. Segundo Bayramoglu et al. (2004) e Merzouk et al. (2010) a eficiência de remoção dos poluentes e o custo operacional relacionam-se diretamente com a condutividade da solução. A solução deve ter um valor de condutividade mínimo para a promoção do fluxo de corrente elétrica.

\subsection{Parâmetros monitorados durante a ECF}

Os valores resultantes do monitoramento durante cada experimento encontram-se na Tabela 1.

Tabela 1 - Resultados obtidos referentes aos parâmetros monitorados na ECF

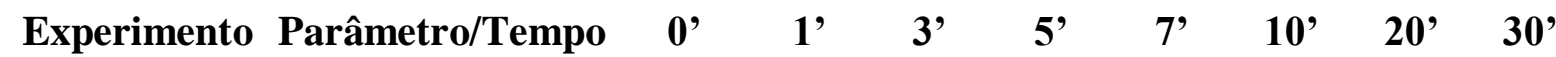

\begin{tabular}{|c|c|c|c|c|c|c|c|c|c|}
\hline \multirow{3}{*}{$\underset{\text { agitação }}{\text { Sem }}$} & $\begin{array}{c}\text { Densidade de } \\
\text { corrente }\left(\mathrm{mA} \cdot \mathrm{cm}^{-2}\right)\end{array}$ & 0 & 1,011 & 1,036 & 1,061 & 1,080 & 1,108 & 1,239 & 1,277 \\
\hline & Temperatura $\left({ }^{\circ} \mathrm{C}\right)$ & 30 & 31 & 32 & 34 & 36 & 38 & 49 & 54 \\
\hline & $\mathrm{pH}$ & 8,70 & 8,71 & 8,73 & 8,94 & 9,12 & 9,32 & 9,49 & 9,59 \\
\hline \multirow{3}{*}{$\begin{array}{l}\text { Agitação de } \\
200 \text { rpm }\end{array}$} & $\begin{array}{c}\text { Densidade de } \\
\text { corrente }\left(\mathrm{mA} \cdot \mathrm{cm}^{-2}\right)\end{array}$ & 0 & 1,123 & 1,14 & 1,151 & 1,173 & 1,198 & 1,291 & 1,328 \\
\hline & Temperatura $\left({ }^{\circ} \mathrm{C}\right)$ & 30 & 32 & 33 & 34 & 36 & 39 & 48 & 53 \\
\hline & $\mathrm{pH}$ & 8,70 & 8,98 & 9,01 & 9,19 & 9,33 & 9,5 & 9,68 & 9,75 \\
\hline \multirow{3}{*}{$\begin{array}{l}\text { Agitação de } \\
700 \text { rpm }\end{array}$} & $\begin{array}{c}\text { Densidade de } \\
\text { corrente }\left(\mathrm{mA} \cdot \mathrm{cm}^{-2}\right)\end{array}$ & 0 & 1,081 & 1,085 & 1,105 & 1,127 & 1,153 & 1,241 & 1,273 \\
\hline & Temperatura $\left({ }^{\circ} \mathrm{C}\right)$ & 30 & 32 & 33 & 35 & 36 & 38 & 47 & 51 \\
\hline & $\mathrm{pH}$ & 8,70 & 9,07 & 9,19 & 9,25 & 9,26 & 9,34 & 9,69 & 9,70 \\
\hline
\end{tabular}


Diante dos resultados apresentados, observou-se que os valores obtidos de densidade de corrente e temperatura aumentaram ao longo do tempo de experimento, para todas as condições de agitação. Nota-se que essas duas variáveis atuam de forma diretamente proporcional, ou seja, quanto maior a temperatura, maior é a atividade das moléculas na solução, como também, maior será a corrente eletrolítica. De acordo com Chen (2004), o aumento da eficiência de corrente com a temperatura foi atribuído ao incremento da atividade de destruição do filme de óxido na superfície do eletrodo.

No tempo total de 30 minutos, os três experimentos obtiveram valores de densidade de corrente e temperatura bastante próximos. Entretanto, o experimento com agitação de $200 \mathrm{rpm}$ apresentou o maior valor de densidade de corrente $\left(1,328 \mathrm{~mA} \cdot \mathrm{cm}^{-2}\right)$, quando comparado aos outros. Em relação à temperatura, o experimento sem agitação alcançou o maior valor $\left(54^{\circ} \mathrm{C}\right)$ no tempo total, podendo ser explicado pela menor homogeneidade no interior do reator, ocorrendo uma menor distribuição de calor na massa líquida.

$\mathrm{O}$ valor do $\mathrm{pH}$ comportou-se de forma semelhante nos três experimentos, aumentando ao longo do tempo de ECF. Esse aumento pode ser atribuído à formação de radicais $\mathrm{OH}^{-}$na reação de redução da água no cátodo, representada pela equação 5:

$$
2 \mathrm{H}_{2} \mathrm{O}+2 e^{-} \rightarrow \mathrm{H}_{2}+2 \mathrm{OH}^{-}
$$

\subsection{Remoção de turbidez}

Como apresentado na Figura 2, os três experimentos de ECF (0, 200 e 700 rpm), mostraram-se bastante eficientes quanto à remoção de turbidez, apresentando resultados acima de 85\% em apenas 5 minutos de reação. O experimento sem agitação destacou-se entre os demais com uma remoção de $96,43 \%$ (5 NTU) com o tempo reacional de 10 minutos, enquanto os experimentos com agitação de 200 e 700 rpm obtiveram, respectivamente, 88,57\% (16 NTU) e 87,86\% (17 NTU) de remoção com o mesmo tempo. Com a promoção da agitação é mais provável que os flocos flotados sejam lançados novamente na solução, reduzindo a eficiência de remoção da turbidez. No tempo total de 30 minutos, os três experimentos obtiveram um percentual de remoção bem próximo, em torno de $97 \%$ (4 NTU). 


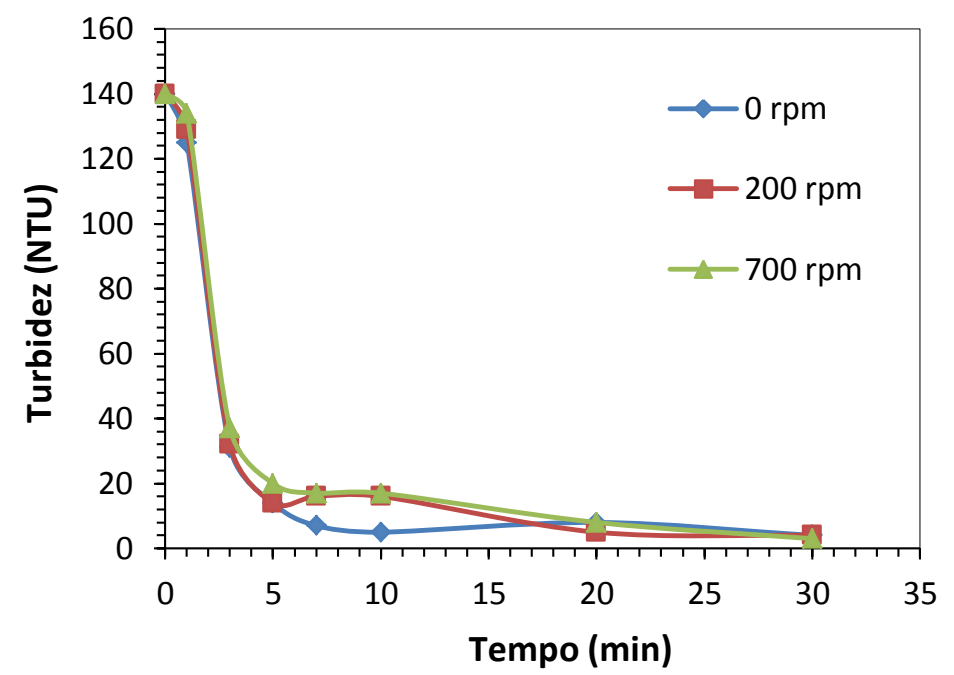

Figura 2 - Cinética de remoção de turbidez segundo o processo de ECF para diferentes velocidades de rotação.

\subsection{Eficiência de descoloração}

Os espectros de UV-vis do efluente bruto e das amostras coletadas do efluente tratado são mostrados na Figura 3. Pode-se observar a inexistência de picos no espectro, provavelmente devido à complexa composição do efluente bruto utilizado nos experimentos, o qual apresenta uma gama de diferentes corantes e outros insumos químicos (peróxido de hidrogênio, cloreto de sódio, ácido acético, dentre outros), característica marcante do efluente final da indústria têxtil que realiza tingimento. Porém, verificou-se um aumento contínuo da absorbância ao longo do intervalo de 360-750 nm. No entanto, é interessante notar que a absorbância global da curva diminui quando os tratamentos de ECF são aplicados. Visualmente isto pode ser observado pela evolução na descoloração das amostras tratadas, conforme estão apresentadas na Figura 4.

Analisando os três experimentos também se notou que a partir de 10 minutos de eletrólise já se obtém uma elevada remoção de cor, tornando-se praticamente estável nos tempos seguintes, com exceção do experimento em que se utilizou a agitação de $700 \mathrm{rpm}$, o qual alcançou uma máxima remoção só após 20 minutos de ECF. A provável causa para essa retardação da descoloração seria a ocorrência da ruptura dos flocos nos quais as moléculas corantes estão agregadas, devido à alta velocidade de agitação. De acordo com Modirshahla et al. (2008), com a elevação na velocidade de agitação até a sua otimização, há um aumento na eficiência de remoção de poluentes. Isto se deve ao fato de que, com a mobilidade dos íons gerados facilitada, os flocos são formados em um menor tempo, no que resulta em um aumento na eficiência de remoção de poluentes durante um tempo de eletrólise em particular. Mas, com uma nova elevação na velocidade de agitação para além do valor ótimo, há a diminuição na eficiência de remoção de poluentes, pois os flocos podem acabar sendo desconstruídos pela colisão de um com o outro, devido à elevada velocidade de agitação. 

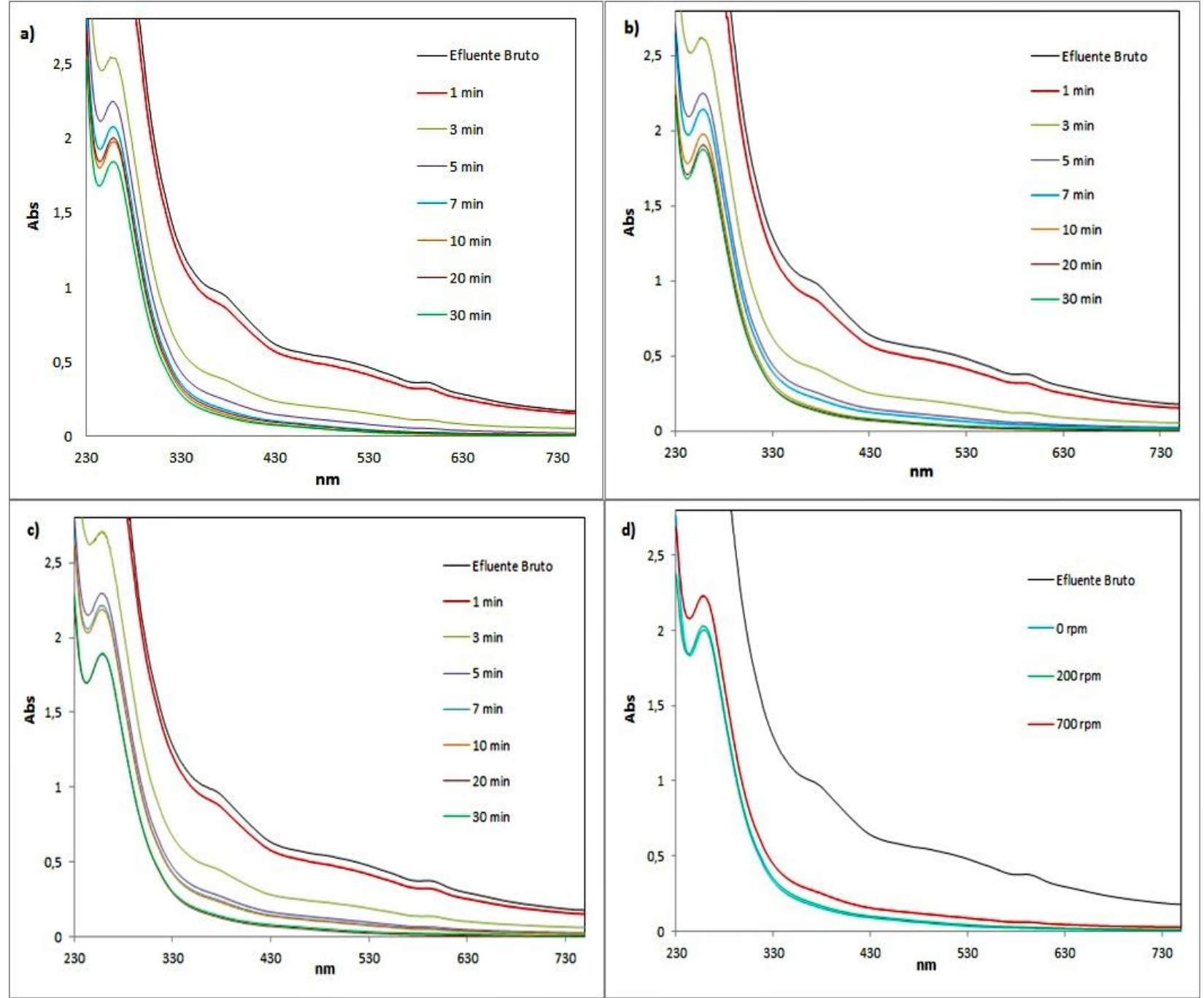

Figura 3 - Espectros de absorbância na região do UV-vis: a) ECF sem agitação; b) ECF com agitação de 200 rpm; c) ECF com agitação de 700 rpm e d) Descoloração após 10 min de ECF nos diferentes níveis de agitação.

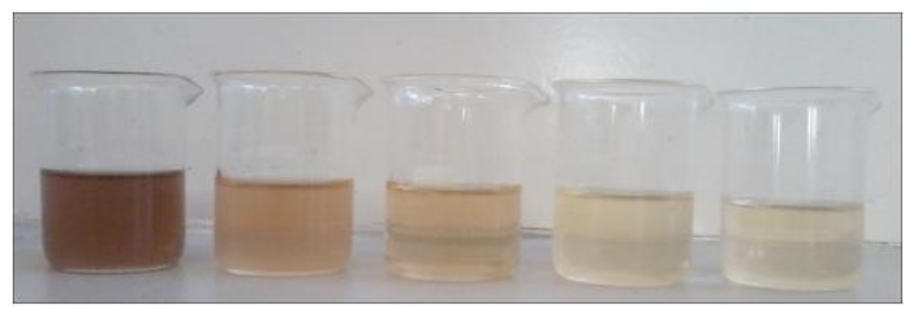


Figura 4 - Amostras do efluente têxtil bruto e tratado por ECF (da esquerda para a direita): efluente bruto, após 3, 5, 10 e $30 \mathrm{~min}$. Velocidade de agitação = $200 \mathrm{rpm}$, densidade de corrente média $=1,2 \mathrm{~mA} \cdot \mathrm{cm}^{-2}$.

\section{CONCLUSÕES}

Conclui-se que o processo de ECF, nas condições experimentadas, proporcionou a clarificação do efluente têxtil utilizado, a partir do tempo ótimo reacional de 10 minutos e sem a necessidade de agitação. Alcançando remoção de turbidez em torno de $96 \%$ e remoção de cor, atestada pela diminuição dos valores da absorbância ao longo da região do UV-vis.

\section{REFERÊNCIAS}

APHA - AWWA - WEF. Standard Methods for the Examination of Water and Wastewater. 21th edition. American Public Health association, American Water Works Association and Water Environment Federation, 2005.

BAYRAMOGLU, M.; KOBYA, M.; CAN, O.T.; SOZBIR, M. Operating cost analysis of electrocoagulation of textile dye wastewater. Sep. Purif. Technol, v. 37 (2), p. 117 e 125, 2004.

CHEN, G. Electrochemical technologies in wastewater treatment. Sep. and Purif. Technol, v.38, p. 11-41, 2004.

HASSEMER, M. E. N.; SENS, M. L.; Tratamento do Efluente de uma Indústria Têxtil. Processo Físico-Químico com Ozônio e Coagulação/Floculação. Rev. Eng. Sanitária e Ambiental, v. 7, p.1, 2002.

HU, C. Y.; LO, S.L.; LI, C. M.; KUAN, W. H. Treating chemical mechanical polishing (CMP) wastewater by electro-coagulation-flotation process with surfactant. J. Hazard. Mater. Amsterdam, v. 120, n. 1, p. 15-20, 2005.

MEDEIROS, A. G. Avaliação dos tratamentos convencional e por oxidação química na degradação de corantes em efluentes têxteis. Dissertação (Mestrado em Engenharia de Processos). Universidade da região de Joinville, UNIVILLE, 2011.

MERZOUK, B.; MADANI, K.; SEKKI, A. Using electrocoagulation and electroflotation technology to treat synthetic solution and textile wastewater, two case studies. Desalination, v 250 (5), p. 573 e $577,2010$.

MODIRSHAHLA, N., BEHNAJADY, M.A., MOHAMMADI-AGHDAM, S. Investigation of the effect of different electrodes and their connections on the removal efficiency of 4-nitrophenol from aqueous solution by electrocoagulation. J. Hazard. Mater, v. 154 (1 e 3), p. 778 e 786, 2008. 